\title{
Neuroimaging Findings as a Predictor of Choice of Antiepileptic Drugs for Seizure Control in West Syndrome
}

\author{
NAZNIN AKTER ${ }^{1 *}$, MUHAMMAD MIZANUR RAHMAN ${ }^{2}$, SHAHEEN AKHTER ${ }^{3}$, KANIJ FATEMA $^{4}$
}

\begin{abstract}
Background: West syndrome (WS) is a severe form of encephalopathy that typically affects young infants ranges from 2-3.5/10,000 live births. Approximately 50\% causes of Infantile Spasm are associated with prenatal factors that includes congenital CNS malformations, intrauterine insults, neurocutaneous syndromes such as Tuberous sclerosis complex, metabolic disorders or genetic syndromes and have poor prognosis. For early identification of underlying etiology a proper neuroimaging is essential that will also help to predict the outcome. For this reason my study aimed to correlate the presence of neuroradiologic abnormalities and its impact on seizure control in the children with West syndrome.
\end{abstract}

Methodology: This retrospective cross sectional study was carried out in Institute of Pediatric Neurodisorder and Autism (IPNA) in BSMMU. All patients those who were diagnosed as a case of West syndrome in between age of 3 months to 3 years according to ILAE (International League against Epilepsy) during period of June 2013 to May 2015 were included in this study.

Results: Thirty one (31) children with West Syndrome were studied, their mean age of presentation was $18 \pm 10.48$ months, mean age of onset of seizure was $6.68 \pm 3.9$ months, $67.7 \%$ of them were male. Patients with normal neuroradiologic findings had early remission of spasms and with anomalies, atrophy and calcification were associated with poor response to drug $(p=0.03)$. ACTH was the most commonly used drug with good seizure remission where neuroimaging finding was normal and in cerebral atrophy as a consequence of perinatal asphyxia $(p=0.06)$. Vigabatrin showed good seizure remission in WS due to Tuberous Sclerosis Complex.

Conclusion: Seizure prognosis of West Syndrome differ greatly according to the type of brain lesion so in every suspected cases of West Syndrome early neuroimaging should have to be performed for choosing an appropriate antiepileptic drug.

Key words: Neuroimaging, West Syndrome

Introduction:

West syndrome (WS) is a severe form of encephalopathy that typically affects young infants and was first described over 160 years ago. ${ }^{1,2}$ The incidence of West syndrome ranges from 2-3.5/10,000

1. Assistant Professor, Department of Pediatrics, Dhaka Medical College

2. Professor \& Chairman of Department of Pediatric Neurology, BSMMU, Dhaka

3. Professor, Department of Pediatric Neurology, BSMMU, Dhaka

4. Associate Professor, Department of Pediatric neurology, BSMMU, Dhaka

Correspondence: Dr. Naznin Akter, Assistant professor, Department of pediatrics, Dhaka Medical College, E-mail: nazninruby73@gmail.com

Received: 27 August 2017

Accepted: 13 March 2018 live births, with onset during the first year of life in $90 \%$ of those affected, ${ }^{2,3}$ this age dependent epileptic syndrome caused by various brain disorders, traditionally it has been classified in to symptomatic, cryptogenic and idiopathic group, but recently this etiological concept has been changed and symptomatic group now recognized as structural/ metabolic, cryptogenic in to unknown and idiopathic become genetic group. ${ }^{4,5,6}$ The prognosis and evolutional changes of WS have usually been discussed according to this classification. Because the symptomatic or structural/metabolic WS group consists of patients with numerous type of brain anomalies and their prognosis and evolutional changes 
varied among the different type of anomalies. Outcomes are usually more favorable among children with cryptogenic/unknown and idiopathic/genetic West syndrome than symptomatic West syndrome. .,7,8 $^{2}$ Symptomatic Infantile Spasm usually present with developmental delay during onset of spasm. Percentage of symptomatic group is now being increasing due to improved diagnostic procedure, such as metabolic and genetic testing and neuroimaging. Approximately $50 \%$ causes of Infantile Spasm associated with a prenatal factors that includes congenital CNS malformations, intrauterine insults, neurocutaneous syndromes such as Tuberous sclerosis complex, metabolic disorders or genetic syndromes such as Down syndrome. 2,9,10 The prognosis of infantile spasm depends on many factors including the underlying etiology, pattern of electroenchephalography, rapidity of response of treatment. Treatment options of infantile spasm included $\mathrm{ACTH}$, prednisolone, vigabatrin and other antiepileptics including sodium valproate, topiramate, levetiracetam etc. Response to ACTH and steroid is varied from $54.5 \%$ to $52.9 \%$ among the symptomatic group and vigabatrin respond well in patient with tuberous sclerosis complex ${ }^{11}$. For early identification of underlying etiology a proper neuroimaging is essential, it provides us detailed information about brain lesions and it will help us to counsel the parent about the prognosis of this syndrome. For this reason my study aimed to correlate the presence of neuroradiologic abnormalities and its effect on seizure control in the children with West syndrome.

\section{Methodology:}

This retrospective cohort study was carried out in the inpatient and outpatient in Institute of Pediatric Neurodisorder and Autism (IPNA) in Banghabandhu Sheikh Mujib Medical University (BSMMU). Shahbagh, Dhaka. All patients those who were diagnosed as a case of West syndrome in between age of 3 months to 3 years according to ILAE (International League against Epilepsy) included in this study. A total of 31 patients were matched the inclusion criteria with required follow up for at least one year. Patients with incomplete records and follow up were excluded from study. After inclusion in to the study proper history of seizures, associated neuro-developmental problems, pregnancy and birth related problems, history of early development, immunization, family and socioeconomic information were collected from the hospital records. The electroencephalographic and neuroimaging either CT scan or MRI of brain findings were collected. Their treatment history was also noted here. Seizure remission rate was calculated and expressed in percentage \% from the baseline number of episode of seizure in 24 hours.

The data were collected by a preformed semistructured questionnaire and entered into Excel for preliminary checking and editing. After necessary works, the data file was transported to SPSS software (statistical package for social sciences, version 21) for final analysis.

Qualitative variables were presented in frequency and percentage while the quantitative data were presented in mean, standard deviation (SD), minimum (Min) and maximum (Max) score of the related variables. The qualitative assessment was done with chi-square test. A p value of $\leq 0.05$ is considered significant. At every step of data collection, processing, data entry and analysis monitoring was done by double checking.

\section{Results:}

A total 31 diagnosed cases of West syndrome were included in this study. Most of the patients were male 21(67.7\%). Maximum patients were between 6-24 months with mean age being $18 \pm 10.48$ months. Mean age of onset of seizure is $6.68 \pm 3.9$ months.

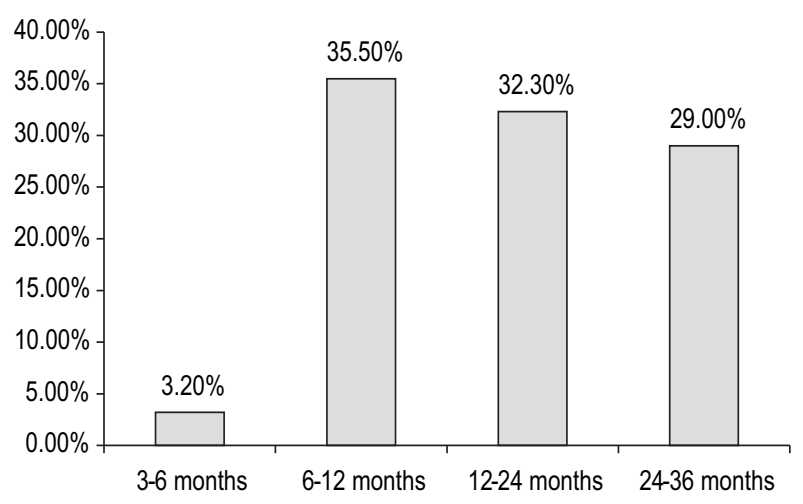

Fig.-1: Frequency of age distribution of child

*Mean age $=(18.00 \pm 10.49)$ months, Age range (4.536) months 


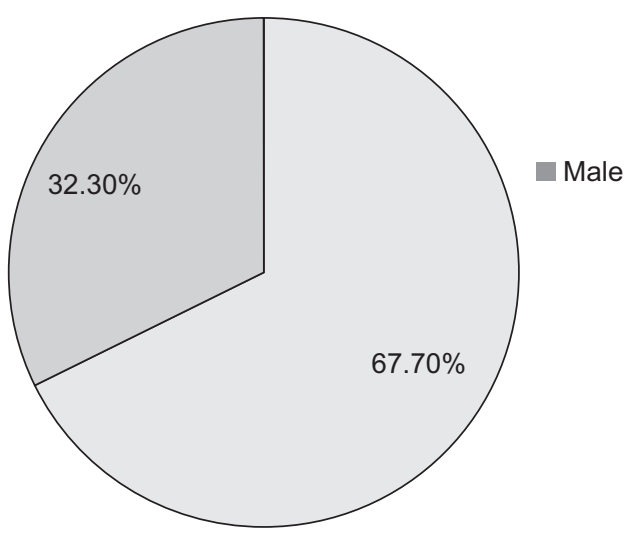

Fig.-2: Frequency of sex distribution of child

Among 31 patients of West syndrome 21(67.70\%) were male child.

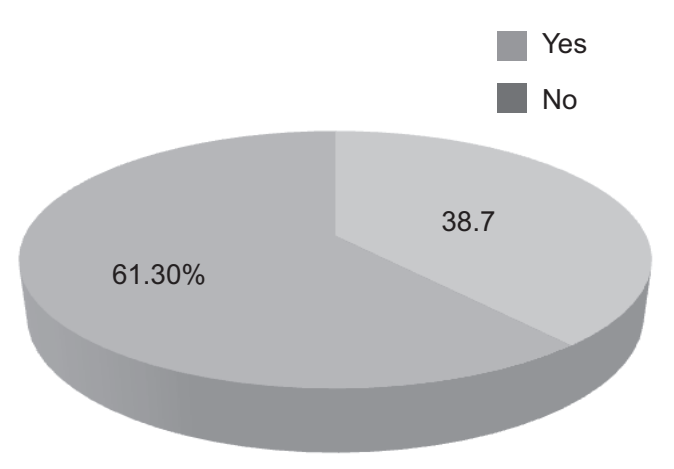

Fig.- 3: Frequency distribution of perinatal asphyxia

Perinatal asphyxia that was evident by history of delayed crying, here this history was found among $12(38.7 \%)$ children.

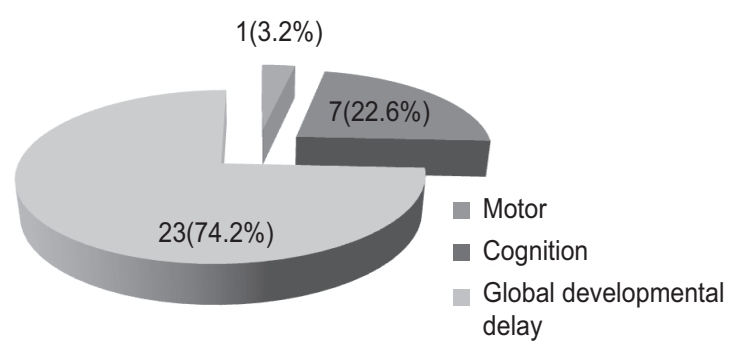

Fig.-4: Frequency distribution of developmental delay

Developmental delay is one of the component of West syndrome, maximum of patients came with global developmental delay, only $7(22.6 \%$ ) were presented with only cognitive delay and one had history of motor delay that manifested as delayed walking.
Table -I

Number of patients per diagnostic group

\begin{tabular}{llc}
\hline Etiologic group & Number of patient \\
\hline 1. & Cryptogenic & 2 \\
2. & Symptomatic & 29 \\
- & Perinatal asphyxia & 12 \\
- & Tuberous sclerosis & 10 \\
- & Sturge-weber syndrome & 1 \\
- & Non. CNS anomalies & 3 \\
\hline
\end{tabular}

29(93.5\%) patients were included in symptomatic group. Only 2 cases, where no definite diagnosis could be made were included in cryptogenic group. The largest group consisted of children with perinatal asphyxia. 10 children were diagnosed as Tuberous sclerosis.

Table-II

EEG abnormalities of studied population

\begin{tabular}{lc}
\hline EEG abnormalities & $\mathrm{N}(\%)$ \\
\hline Hysparrythmia & $17(54.8)$ \\
Epileptic enchephalopathy & $10(32.3)$ \\
Focal epileptic discharge & $4(12.9)$ \\
\hline
\end{tabular}

Thirty one (31) children with Infantile spasms presented with EEG abnormalities but typical hypsarrhythmiawas present on EEG in $17(54.8 \%)$ patients, $10(32.3 \%)$ had features of epileptic encephalopathy and $4(12.9 \%)$ had focal epileptic discharge on EEG.

Table-III

Neuroimaging abnormalities of studied population

\begin{tabular}{lc}
\hline Abnormal neuroimaging findings & Frequency $(\%)$ \\
\hline Congenital malformation & $3(9.7)$ \\
Lissencephaly & 1 \\
Closed lip schizencephaly & 1 \\
Focal cortical dysplasia & 1 \\
Atrophy & $14(45.16)$ \\
Perinatal asphyxia & 12 \\
Glutaricaciduria & 1 \\
Non ketotichyperglycinemia & 1 \\
Calcification & $11(35.5)$ \\
Tuberous sclerosis complex & 10 \\
Sturge weber symdrome & 1 \\
Hyperintense signal & $1(3.2)$ \\
Normal & $2(6.5)$ \\
\hline Total & $31(100)$ \\
\hline
\end{tabular}

Most common findings on Neuroimaging that observed were cortical atrophy and the second common Neuroimaging abnormalities were intracranial calcification secondary to neurocutaneous syndrome. 
Table-IV

Rate of seizure remission with antiepileptic drugs among studied population

\begin{tabular}{|c|c|c|c|c|c|c|}
\hline \multirow[t]{2}{*}{ Antiepileptic drugs } & \multicolumn{4}{|c|}{ Seizure remission } & \multirow[t]{2}{*}{ Total } & \multirow[t]{2}{*}{$p$} \\
\hline & No $(0 \%)$ & $25-<50 \%$ & $50-<75 \%$ & $75-100 \%$ & & \\
\hline$\overline{\mathrm{ACTH}}$ & 0 & $2(16.6)$ & $5(41.7)$ & $5(41.7)$ & 12 & 0.06 \\
\hline Vigabatrin & 0 & 0 & $4(66.7)$ & $2(33.3)$ & 6 & \\
\hline Steroid & 0 & $1(25.0)$ & $1(25.0)$ & $2(50.0)$ & 4 & \\
\hline Other+ combined & $4(44.4)$ & 0 & $1(11.2)$ & $4(44.4)$ & 9 & \\
\hline$\overline{\text { Total }}$ & $4(12.9)$ & $3(9.7)$ & $11(35.5)$ & $13(41.9)$ & 31 & \\
\hline
\end{tabular}

ACTH was the most common drug that used to treat Infantile Spasm, seizure remission was also good compared to other medication like Vigabatrin, steroid and other medication like Na-valproate, Levetiracetam, Topiramate.

Table - V

Correlation of abnormal neuroimaging findings with seizure remission among studied population

\begin{tabular}{lcccccc}
\hline Abnormal neuroimaging findings & \multicolumn{4}{c}{ Percentage of seizure remission } & Total & $p$ \\
\cline { 2 - 6 } & No & $25-<50 \%$ & $50-<75 \%$ & $75-100 \%$ & & \\
\hline Congenital malformation $^{*}$ & $2(66.7)$ & 0 & $1(33.3)$ & 0 & 3 & $0.03^{*}$ \\
Atrophy* $^{*}$ Calcification $^{*}$ & $1(6.7)$ & $2(13.3)$ & $6(40.0)$ & $6(40.0)$ & 15 & \\
Hyperintense signal & $1(10.0)$ & 0 & $5(45.0)$ & $5(45.0)$ & 10 & \\
Normal & 0 & $1(100)$ & 0 & 0 & 1 & \\
\hline Total & 0 & 0 & 0 & $2(100)$ & 2 & \\
\hline
\end{tabular}

We compare the abnormal neuroimaging findings with the seizure outcome and found it statistically significant that mean abnormal neuroimaging findings like congenital malformation are associated with poor seizure control calcification secondary to TSC have shown good seizure remission.

\section{Discussion}

This retrospective chart analysis of neuroimaging findings and its role to identify etiology and its relation with seizure outcome for infantile spasm have found male predominance $(67.7 \%)$ among the studied population which is comparable to other study. ${ }^{11,12}$ Mean age of seizure onset is $6.68 \pm 3.9$ months, which is similar to the findings of study done by Betul Tekin Guvoli et al. ${ }^{13}$

The cause of infantile spasm related to perinatal asphyxia was $38.7 \%$ in this study, which is similar to the studies done by other developing countries. ${ }^{11}$ Global developmental delays was the most common association presented among $74.2 \%$ of studied population.
Results of different published studies have shown that $70 \%$ of patients with infantile spasm have identifiable etiology on neuroimaging. ${ }^{14}$ In this study $93.5 \%$ had identifiable etiology according to majority they are perinatal asphyxia and neurocutaneous syndrome.

EEG was done to all studied population only $54.8 \%$ have shown typical hysparrhythmia, next common findings of EEG was epileptic encephalopathy. Modified hysparrhythmia, multifocal discharge, burst suppression patterns are other change of EEG in infantile spasm which was not present among our studied population. ${ }^{11,12}$ Among 14 (38.7\%) patients with cortical atrophy on neuroimaging 12 of them had history of delayed crying. Intracranial calcification was detected on $11(32.3 \%)$ cases, among them 10 were diagnosed as Tuberous Sclerosis Complex (TSC), One was Sturge-Weber-Syndrome. Three diagnosed cases of metabolic syndrome were presented with Infantile Spasm, one was Glutaricaciduria Type1, showed typical batwing appearance on MRI of brain, another one was Maple Syrup Urine Disease(MSUD) 
which had hyperintense signal on T2 image and features of demyelination on MRI , last case of metabolic syndrome was non ketotichyperglycinemia which had generalized cortical atrophy on MRI, these all three Infantile Spasm with metabolic disorder was medically intractable. Three patients had congenital malformations of brain on neuroimaging,they were lissencephaly, closed lip schizencephaly and focal cortical dysplasia. Lissencephalic and schizencephalic patients seizure was medically intractable, patients with focal cortical dysplasia had shown $55 \%$ seizure remission rate. In this study cessation of spasm was regarded as the most important outcome measurement, for this reason its very important to select a uniform treatment protocol but we failed to do so, because in our centre we have no strict protocol to treat IS. In this study we found Inj. ACTH was used most, next common drug was vigabatrin specially in patients with TSC, third common used drug was steroid; Na-valproate, Levetiracetam, Topiramate, Nitrazepam was also used as add on drug to control refractory spasm. Among these above mentioned drug ACTH had shown better seizure remission. Finally we compare the neurological abnormalities with seizure remission and it was found statistically significant $(p=0.03)$ result that is congenital malformation, had least seizure remission whereas 2 patient with normal neuroimage findings have shown $100 \%$ seizure remission. Although this difference in seizure remission could also be explained by the treatment choice but still different study have shown better outcome in ACTH compared to vigabatrin ${ }^{15,16}$. In our study vigabatrin was the initial choice for IS caused by TSC, it also showed good response for seizure remission.

\section{Conclusion:}

Neuroimage has a well established role to identify the etiology of infantile spasm. Early initiation of appropriate drugs according to etiology of infantile spasm will help to improve seizure remission and will also help to counsel the parents regarding its outcome.

\section{Recommendation:}

In every diagnosed cases of West syndrome a neuroimaging should have to be done at early stage for making a proper management plan and for proper counselling of parents about the outcome.

\section{Limitations of the study:}

Retrospective study with small sample size from a single centre.

\section{References:}

1. Kenneth F. Swaiman S A, Donna M F, Nina F.S,Myoclonic Seizures and Infantile Spasms, Swaiman's Pediatric Neurology Principles and Practice, fifth edition, Elsevier, 2011; Chapter 56: p781-789.

2. James WW, Patricia A G, Rosbeck K L, Hardin M, O' DellC, Whittemore $\mathrm{V}$ and Pellock J M, Infantile spasms (west syndrome): Update and resources for pediatricians and providers to share with parents. BioMedCentre Pediatrics 2012; 12 : 108.

3. Okumura A, Watanabe K, Negoro T, AsoK, Natsumo J, Matsumoto A et al, Evolutional changes and outcome of west syndrome: Correlation with Magnetic Resonance Imaging findings. Epilepsia1998; 39(suppl. 5): 46-49.

4. Anne T, Berg and Ingrid E, Scheffer, New concept in classification of epilepsies: Entering the $21^{\text {st }}$ century. Epilepsia, 2011.52(6): 1058-1062.

5. Commision on classification and Terminology of the International League Against Epilepsy. Proposal for revised clinical and electrographic classification of epileptic seizures. Epilepsia 1981, 22: 489-501.

6. Commision on Classification and Terminology of the International League Against Epilepsy. Proposal for revised classification of epilepsies and epilepsy syndromes. Epilepsia 1989; 30: 389-399.

7. Wong $M$, Trevathan E: Infantile Spasms. PediatrNeurol 2001, 24:89-98.

8. Lombroso CT. A prospective study of infantile spasms: Clinical and therapeutic correlates. Epilepsia 1983; 24: 135-158.

9. JellingerK :Neuropathological aspects of Infantile Spasms. Brain Dev 1987, 9: 349-357.

10. Paciorkowski AR, Thio LL, Dobyns WB: Genetic and Biologic Classification of Infantile Spasms. PediatrNeurol 2011, 45: 355-367. 
11. Aydini N, Caliskan M, Ozmen M, Tonguc E. Neuroradiologic aspects of West syndrome. Pediatric neurology 1998; 19(3): 211-216.

12. KarlaV, Gulati S, MohonR P, MenonS. West syndrome and other infantile epileptic encepalopathies - Indian hospital experience. Brain \& Development, 2002; 24: 130-139.

13. Guveli BT, Coker O, Dortcan N, Benbir G, Dervent A. Long term outcome in patients with west syndrome: An outpatient clinical study. Seizure, 2015;25: 68- 71.
14. Matsumoto A, Watanabe $\mathrm{K}$ et al. Prognostic factors of infantile spasms from the etiological view point. Brain Dev 1981; 3: 361-4.

15. Lieven L, Helene V, Berten C. Treatment and long term and long term outcome in West syndrome: The clinical reality. A multicentre follow up study. Seizure 2010; 19: 159-164.

16. Lux AL, Osborne JP. The influence of etiology upon ictal semiology, treatment decisions and long term outcomes in infantile spasms and West syndrome. Epilepsy Research 2006; 70: 77-86. 